\title{
Dihydromyricetin protects against cerebral ischemia/reperfusion injury via suppressing microglia-mediated neuroinflammation and activation of ERK1/2-CREB signaling pathway
}

Yafei Zhao ${ }^{\mathrm{a}, \mathrm{b}, *}$, Panpan Wang ${ }^{\mathrm{a}, \mathrm{b}, *}$, Shuangshuang Chen ${ }^{\mathrm{a}, \mathrm{b}}$, Chaojun Han $^{\mathrm{a}, \mathrm{b}}$, Qiuting Yan ${ }^{\mathrm{a}, \mathrm{b}}$, Longtai Zheng ${ }^{\mathrm{a}, \mathrm{b}}$, Jia Jia ${ }^{\mathrm{a}, \mathrm{b}}$, Zhaoxiang Ren ${ }^{\mathrm{a}, \mathrm{b}, \#}$, Xuechu Zhen ${ }^{\mathrm{a}, \mathrm{b}, \#}$

${ }^{\mathrm{a}}$ Jiangsu Key Laboratory of Translational Research and Therapy for Neuro-Psycho-Diseases and

College of Pharmaceutical Sciences, Soochow University, Suzhou, Jiangsu 215021, China

${ }^{\mathrm{b}}$ The CollaborativeInnovationCenter for Brain Science, SoochowUniversity, Suzhou, China

*Authors contributed equally to this work

\# Correspondence to: Zhaoxiang Ren or Xuechu Zhen, Department of Pharmacology, College of Pharmaceutical Sciences, Soochow University, Suzhou, Jiangsu 215021, China. Email: hnkfrzx@163.com; zhenxuechu@suda.edu.cn

\begin{abstract}
Dihydromyricetin (DHM), a natural flavonoid compound extracted from the fruit Ampelopsis grossedentata, exerts various pharmacological effects. We explored the neuroprotective effects of DHM following cerebral ischemia. Male ICR mice were intraperitoneally injected with DHM for 7 days. Post-ischemic neurological deficits were evaluated with behavioral tests. Mice brain tissues were harvested for infarction analysis, immunohistochemistry. The production of pro-inflammatory mediators in microglial cells were assessed by qPCR and ELISA. Neuroprotective effects of DHM were assessed in HT22 neuronal cells subjected to oxygen-glucose deprivation (OGD)/reoxygenation. DHM reduced the activation of microglia, protected HT22
\end{abstract}


neurons against OGD-induced injury and promoted functional recovery following middle cerebral artery occlusion (MCAO). Mechanistically, DHM reduced release of pro-inflammatory mediators from microglia. In addition, DHM suppressed caspase-3 cleavage and upregulated CREB, Bcl-2 and phosphorylation of ERK1/2 in HT22 cells. Our study suggested that DHM protects against cerebral ischemia by suppressing microglial neuroinflammation and activating neuronal ERK-CREB-Bcl-2 signaling pathway.

Keywords: Dihydromyricetin (DHM), Neuroinflammation, Microglia, Cerebral ischemia

\section{Introduction}

Ischemic stroke is one of the leading causes of death and disability worldwide, and represents a severe health problem for human beings (Feigin et al., 2015; Meschia et al., 2014). Ischemia and reperfusion (I/R) activates a large number of pathological cascades, such as oxidative stress, inflammation, mitochondrial damage and excitotoxicity (Hacke et al., 2008; X. Hou et al., 2015; Howard, 2010). Particularly, the resident microglia of the central nervous system are activated to release a large number of pro-inflammatory mediators following cerebral ischemia, which significantly contribute to post-ischemic secondary deterioration and tissue damage (Chen et al., 2016; Tao et al., 2014; Taylor \& Sansing, 2013). Thus, attenuating microglia-mediated neuroinflammation represents a promising therapy for ischemic stroke (Denes et al., 2007; Gaire et al., 2015; Weinstein, Koerner, \& Moller, 2010). ERK1/2 are important members of mitogen-activated protein kinases (MAPKs) family that regulate cell survival and apoptosis (Sebastien Cagnol \& Chambard, 2009; S. Cagnol \& Chambard, 2010; Yujiri, Sather, Fanger, \& Johnson, 1998). Evidence exists to suggest that activation of ERK signaling pathway contributes to neuroprotection following ischemia injury. The ERK signaling pathway further 
phosphorylates and activates the cAMP-response element binding protein (CREB), which in turn regulates the expression of brain-derived neurotrophic factors (BDNF) and the B cell lymphoma 2 (Bcl-2) (Choi et al., 2013; Sasaki et al., 2011).

Dihydromyricetin (DHM) is a flavonoid compound extracted from the stems and leaves of Ampelopsis grossedentata (Jiang et al., 2015). Recent studies reported that DHM displayed widely protective effects, such as anti-oxidation and anti-inflammatory (X. Hou et al., 2015; X. L. Hou et al., 2015; Ye, Wang, Duncan, Eigel, \& O'Keefe, 2015), anti-cancer (Fan et al., 2016; Liu et al., 2015), improvement of insulin sensitivity (Shi et al., 2015). We have recently shown that DHM reduced dopaminergic neuronal loss in MPTP-induced Parkinson's disease (Ren, Zhao, Cao, \& Zhen, 2016). All these findings suggest that DHM may be a potential food supplement against damage or insult. Here, we further investigated whether DHM protectes against cerebral ischemia injury and its potential mechanism.

\section{Materials and Methods}

\subsection{Chemicals and reagents}

DHM was purchased from Shanghai Standard Biotech Co, Ltd (Shanghai, China). U0126 was purchased from Selleck; antibodies against cleaved caspase-3, CREB, phospho-CREB, Bcl-2 were purchased from Cell Signaling Technology (Danvers, MA, USA); antibodies against COX-2, anti-iNOS, anti-ERK1/2, anti-P65 and anti-phospho-ERK1/2 were purchased from SANTA CRUZ; antibodies against Iba1 and NeuN were purchased from MILLIPORE, antibodies against $\alpha$-tubulin were purchased from Sigma-Aldrich. Primers for qPCR were obtained from Shanghai SangonBiotech Co, Ltd. RT-PCR kit was purchased from Dalian Takara Biotechnology Co, Ltd. TNF- $\alpha$ and IL-6 ELISA kits were purchased from Wuhan Boster Biosciences Co, Ltd.

\subsection{Animals and ischemia/reperfusion model}

Male ICR mice were purchased from SLAC Laboratory Animal (Shanghai, China). Mice were kept in a 12-hour light/dark cycle with ad libitum access to food and water. 
All animals care and experimental procedures were approved by the Animal Care and Use Committee of Soochow University. Focal ischemia was induced according to previous reports (Ni et al., 2015). Mice were anesthetized with isoflurane. A neck incision was made to expose the right common carotid (CCA), external carotid artery (ECA) and internal carotidartery (ICA). A nylon monofilament coated with silicon (diameter $0.22 \pm 0.02 \mathrm{~mm}$ ) was inserted until it reached the origin of the middle cerebral artery. After $1 \mathrm{~h}$ of MCAO, blood flow was restored by withdrawing the monofilament. Mice of sham group were subjected to the identical surgeries except for monofilament insertion.

\subsection{Animal groups and DHM administration}

DHM was dissolved in dimethylsulfoxide (DMSO) to a concentration of $0.5 \mathrm{mg} / \mu \mathrm{L}$, and diluted with saline for intraperitoneal injection $(10$ or $20 \mathrm{mg} / \mathrm{kg}$ ). The final DMSO concentration was below $0.1 \%$, and the injection volume was $1 \mathrm{~mL} / \mathrm{kg}$ of body weight. Mice were randomly divided into four groups, Sham-operated group, MCAO groups were treated with saline (vechicle), DHM (10 mg/kg) or DHM (20 $\mathrm{mg} / \mathrm{kg}$ ), respectively. Mice were injected with 10 or $20 \mathrm{mg} / \mathrm{kg}$ DHM daily for 7 days: 3 days before MCAO and 4 days following MCAO procedure. MCAO mice injected with DHM are compared with vechicle.

\subsection{Behavioral testing}

\subsection{1. mNSS test}

The modified neurological severity scores (mNSS) was graded on a scale of 0-18 as previously described. Briefly, mNSS was used to assess motor function, sensory function, balance and reflex after ischemia/reperfusion. The more scores were awarded, the more serious damage was (Jin et al., 2014).

\subsubsection{Corner test}

As the previous reported, sensorimotor abnormalities after MCAO were assessed using a corner which consisted of two cardboard pieces $\left(30 \times 20 \times 1 \mathrm{~cm}^{3}\right)$. Mice were 
forced into the corner and both sides of vibrissae were stimulated, which resulted in mice rear and turn back to the open end. Mice preferentially turned toward the impaired side following MCAO. By contrast, the probability for sham mice to turn left or right is almost equal. Numbers of left or right turns were recorded over ten trials (Jin et al., 2014).

\subsubsection{Rotarod test}

Mice were placed on the rotarod and the speed was increased from 5 to $40 \mathrm{r} / \mathrm{min}$ in 5 min. The time was recorded when mice fell off the rotarod. Each mouse was tested for three trials with a 30 min interval. Results were averaged for three trails (Jin et al., 2014).

\subsection{Analysis of brain infarction}

At $96 \mathrm{~h}$ after reperfusion, mice were sacrificed. The whole brains were harvested, and sectioned into four consecutive coronal slices and incubated in saline containing $2 \%$ 2,3,5-triphenyltetrazolium chloride (TTC) at $37{ }^{\circ} \mathrm{C}$ for 10 minutes and subsequently fixed in $4 \%$ paraformaldehyde overnight. The ImageJ software (Sigma Scan Pro, Jandel, San Rafael, CA) was used to measure the infarct volumes.

\subsection{Immunocytochemistry}

BV2 cells were seeded on glass coverslips in 12-well plates and cultured overnight. The cells were treated with $50 \mu \mathrm{M}$ DHM for $4 \mathrm{~h}$ before stimulated with $200 \mathrm{ng} / \mathrm{ml}$ LPS. The cells were fixed in $4 \%$ cold paraformaldehyde for 30 min, rinsed with PBST (PBS containing $0.3 \%$ Triton) three times and then blocked with $0.3 \%$ Triton $\mathrm{X}-100$ and 3\% BSA in PBS for $60 \mathrm{~min}$. The cells were subsequently incubated with antibodies against P65 (1:300; Santa Cruz, CA, USA) overnight at $4^{\circ} \mathrm{C}$. Next, the cells were rinsed 30 min with PBST and incubated sequentially with Alexa Fluor 488 (green)-conjugated anti-rabbit IgG antibodies (1:1000; Cell signaling) for $2 \mathrm{~h}$ and Hoechst (1:10,000; Invitrogen, Carlsbad, CA, USA) for $30 \mathrm{~min}$. Cell immunofluorescence images were captured with a laser confocal fluorescent 
microscope (LSM 700; Zeiss, Oberkochen, Germany).

After assessment with behavioral tests, mice were anesthetized and perfused with saline followed by $4 \%$ paraformaldehyde. The whole brains were collected and post-fixed in paraformaldehyde overnight at $4^{\circ} \mathrm{C}$. The brains were serially cut into 20 mm thick coronal sections. The sections were blocked with PBS containing 3\% BSA and $0.3 \%$ Triton for $1 \mathrm{~h}$. Then the sections were incubated overnight at $4^{\circ} \mathrm{C}$ with the following primary antibodies: rabbit anti-Ibal (1:200) or rabbit anti-NeuN (1:300). Then, the sections were rinsed with PBST and incubated with Alexa Fluor 594 conjugated goat anti-rabbit IgG (1:400, Thermo Fisher Scientific) or Alexa Fluor 488 conjugated goat anti-goat $\operatorname{IgG}(1: 400$, Thermo Fisher Scientific) for $2 \mathrm{~h}$ at room temperature. After mounting, the sections of ischemic lesions were obtained by using a laser confocal fluorescent microscope (Wang et al., 2016).

\subsection{Cell culture}

Murine BV2 microglial cells and murine hippocampal neuronal HT22 cell lines were cultured with complete DMEM medium (containing 10\% fetal bovine serum (FBS) and $1 \%$ penicillin/streptomycin) at $37^{\circ} \mathrm{C}$ in a $5 \% \mathrm{CO} 2$ incubator.

\subsection{NO determination}

The level of NO produced by BV2 cell was assessed with Griess reagent. Briefly, BV2 cells were seeded in 96-well plates at a density of $1 \times 10^{5} / \mathrm{cm}^{2}$. Cells were treated with LPS (200 ng/ml, Sigma) with or without DHM for $24 \mathrm{~h}$. Then, BV2 supernatant $(50 \mu \mathrm{L})$ was transferred into another 96-well plate and mixed with $50 \mu \mathrm{L}$ Griess reagent. Finally, the absorbance was assessed at $540 \mathrm{~nm}$ on a Microplate Reader (Multiskan MK3; Thermo Scientific).

\subsection{Measurement of TNF- $\alpha$ and IL-6 with enzyme-Linked immunosorbent assay}

\section{(ELISA)}

Bv2 cells $\left(2 \times 10^{5}\right.$ cells/well $)$ were seeded in 12 -well plates. After $12 \mathrm{~h}$, cells were treated with $200 \mathrm{ng} / \mathrm{mL}$ LPS alone or with serial concentrations of DHM. After 
another $24 \mathrm{~h}$, the supernatans of BV2 containing LPS and DHM were collected, then the concentrations of TNF- $\alpha$ and IL-6 were measured with commercial ELISA kits (Boster Biosciences Co, Wuhan, China) according to the manufacture's protocol.

\subsection{Apoptosis detection}

BV2 cells were pretreated with DHM 30 min prior to LPS treatment for additional 12 h. Then, the supernatans containing LPS and DHM were removed and added fresh medium to BV2 cells, cultured for $24 \mathrm{~h}$. Finally, supernatans were added to HT22 cells. After $24 \mathrm{~h}$ treatment, flow cytometry were used to assess apoptosis with an Annexin V-FITC Apoptosis Detection Kit (Beyotime, Shanghai, China). Briefly, cells were harvested, re-suspended in $195 \mu \mathrm{L}$ of Annexin V-FITC combing solution and incubated in $10 \mu \mathrm{L}$ of propidium iodide and $5 \mu \mathrm{L}$ of Annexin V-FITC in the dark at room temperature for $20 \mathrm{~min}$. Subsequently, the solution was analyzed with flow cytometry.

\subsection{Exposure to oxygen-glucose deprivation (OGD)}

HT22 cells were seeded in 96-well culture plates and cultured overnight. Then, the medium was replaced with OGD buffer ( Nacl: $4.09 \mathrm{~g}, \mathrm{Kcl} ; 186.5 \mathrm{mg}, \mathrm{Cacl}_{2}: 111 \mathrm{mg}$, HEPES: $2.38 \mathrm{~g}$ and Glycine: $1.125 \mathrm{~g}$, dissolved in $500 \mathrm{ml}$ double distilled water) and cells were exposed to oxygen-deprivation for 6-8 h. During OGD, the cells were treated with different concentrations of DHM. After OGD, OGD buffer was replaced with complete DMEM medium and cultured at $37{ }^{\circ} \mathrm{C}$ in a $5 \% \mathrm{CO} / 95 \%$ incubator as previously described (Ni et al., 2015).

\subsection{Cell viability assay}

HT22 cells were seeded in 96-well plates at the density of 5000 cells per well and cultured overnight. Then, cells were subjected to OGD/reoxygenation and treated with different concentrations of DHM or vehicle, as described above. Cell viability was assessed with MTT. Briefly, each well was added with $30 \mu \mathrm{L}$ of MTT solution $(0.5$ $\mathrm{mg} / \mathrm{mL}$ ). The plate was incubated for $2 \mathrm{~h}$ at $37^{\circ} \mathrm{C}$, then $100 \mu \mathrm{L}$ DMSO was added to 
each well. Finally, the absorbance at $570 \mathrm{~nm}$ was assessed using a Microplate Reader (Multiskan MK3; Thermo Scientific).

\subsection{Real-time PCR}

Total RNA was isolated from the BV2 cells by using Trizolreagent (Takara, Shiga, Japan). RNA (1 $1 \mu \mathrm{g})$ was reversely transcribed using murine leukemia virus reverse transcriptase and oligo (dT) as the primer according to the manufacturer's protocol and as described before (Gan et al., 2015). GAPDH was measured as the internal control, qPCR was performed using SYBR Premix II (Bio-Rad, Hercules, CA, USA). The parameters of qPCR: incubation at $50{ }^{\circ} \mathrm{C}$ for $2 \mathrm{~min}$, denaturing at $95{ }^{\circ} \mathrm{C}$ for $10 \mathrm{~min}$, followed by 40 cycles of $95^{\circ} \mathrm{C}$ for $15 \mathrm{~s}$ and $60{ }^{\circ} \mathrm{C}$ for $1 \mathrm{~min}$. Specific qPCR primers were as follows: iNOS, Forward primer: 5'-CAGGAGGAGAGAGATCCGATTTA-3'; Reverse primer: 5'-GCATTAGCATGGAAGCAAAGA-3'; COX-2, Forward primer: 5'-CAG GCT GAA CTT CGA AAC A-3'; primer: 5'-GCT CAC GAG GCC ACT GAT ACC TA-3'; $\quad$ TNF-a, Forward primer: 5'-CATCTTCTCAAAATTCGAGTGACAA-3'; primer: 5'-TGGGAGTAGACAAGGTACAACCC-3'; IL-1 $\beta$, Forward primer: 5'-TCCAGGATGAGGACATGAGCAC-3'; primer: 5'-GAACGTCACACACCAGCAGGTTA-3'; IL-6, Forward primer: 5'-TTCCATCCAGTTGCCTTCTT-3'; Rrimer: 5'-CAGAATTGCCATTGCACAAC-3'; GAPDH, Forward primer: 5'-ACCACAGTCCATGCCATCAC-3'; Rrimer: 5'-TCCACCACCCTGTTGCTGTA-3';

\subsection{Western blot}

BV2 or HT22 cells were lysed with RIPA buffer (Cell Signaling Technology, USA) for $30 \mathrm{~min}$. Protein concentration was determined with a protein assay kits (Bio-Rad, Hercules, CA, USA). Forty micrograms of protein were separated on $8 \%$ sodium dodecyl sulfate/polyacrylamide gel electrophoresis gels and then transferred to polyvinylidenedifluoride membranes (Millipore, Bedford, MA, USA). After blocking 
in $5 \%$ skim milk for $1 \mathrm{~h}$, the membranes were incubated with the following primary antibodies: anti-cleaved caspase-3 (1:500), anti-COX-2 (1:1000), anti-iNOS (1:1000), anti-ERK1/2 (1:1000), anti-phospho-ERK1/2 (1:1000), anti-CREB (1:1000), anti-phospho-CREB (1:1000), anti-Bcl-2 (1:800) and anti- $\alpha$-tubulin (1:10000). Then, membranes were incubated with secondary antibodies (Sigma-Aldrich). The results were analyzed using a ChemiScope 3300 Mini (Clinx Science Instruments, Shanghai, China).

\subsection{Statistical analysis}

Data were expressed as the standard error of the (SEM) and analyzed with the GraphPad software (Version 6.0). One-way ANOVA followed by Bonferroni post hoc test was utilized for multiple-group comparisons. $\mathrm{P}<0.05$ was considered statistically significant.

\section{Results}

3.1. DHM reduces infarct volumes and improves functional recovery in mice following MCAO

Compared to vehicle, administration of DHM at $10 \mathrm{mg} / \mathrm{kg}$ and $20 \mathrm{mg} / \mathrm{kg}$ remarkably decreased infarct volumes by $16 \%$ and $25 \%$, respectively (Fig. 1A, B). Moreover, DMH-treated mice exhibited significant improvement in three behavioral tests (Fig. 1C, 1D and 1E), suggesting that DHM treatment promoted functional recovery following MCAO.

\subsection{DHM suppresses microglial activation and improves neuronal survival}

Immunohistochemical analyses showed that the number of NeuN-positive cells in the ipsilateral cortical regions of MCAO mice was markedly increased by DHM treatment $(10 \mathrm{mg} / \mathrm{kg}, 20 \mathrm{mg} / \mathrm{kg})$ as compared with that in vehicle groups (Fig. 2A). Meanwhile, the number of Iba1-postive cells was significantly increased in ipsilateral cortical regions measured at 4 days after MCAO, which was attenuated in mice treated with $10 \mathrm{mg} / \mathrm{kg}$ or $20 \mathrm{mg} / \mathrm{kg}$ DHM (Fig. 2B). These data suggested that DHM 
effectively suppressed microglial activation and improved neuronal cells survival in the ischemic brains.

\subsection{DHM attenuates inflammatory responses of LPS-activated BV2 microglia}

To further elucidate the effect of DHM on microglial activation, we employed microglial BV2 cell line to study the inhibitory effect of DHM on microglia-mediated inflammation. As shown in Fig. 3A, DMH did not affect the viability of BV2 cells at the concentrations ranging from 0 to $50 \mu \mathrm{M}$ (Fig. 3A). DHM decreased LPS-induction of nitrite production (Fig. 3B). In addition, DHM reduced LPS-stimulated mRNA expression of COX-2, iNOS, TNF- $\alpha$, IL-6 and IL- $\beta$ in BV2 cells (Fig. 3E). At the protein level, DHM also effectively reversed LPS-induction of the inflammatory mediators COX-2, iNOS and pro-inflammatory cytokines TNF- $\alpha$ and IL-6 in a concentration-dependent manner (Fig. 3C and 3D). The translocation of NF-kB (p65) to the nucleus was obviously inhibited by DHM in LPS-treated BV2 cells (Fig. 3F). All these data demonstrated that DHM elicited a potent inhibition on microglial activation.

\subsection{DHM protects HT22 cells from apoptosis induced by microglia-mediated neurotoxicity}

It is well-known that overactivation of microglia results in neuronal damage. We next tested the protective effect of DHM-inhibited microglial activation on neuronal protection. In agreement with previous observation (Wu et al., 2015), the conditioned media (CM) collected from LPS-activated microglia significantly induced apoptosis of HT22 neuronal cells, However, the CM from DHM-treated microglial cells attenuated microglia-mediated neurotoxicity induced by LPS (Fig. 4). The data indicated that DHM-inhibited microglial activation contributes to neuronal survival.

\subsection{DHM protects OGD/ reperfusion-induced neuronal cell death}

To elucidate if DHM elicits direct neuronal protection, we employed OGD approach to test the effect of DHM. As shown in Fig. 5, 8 hours of OGD significantly induced 
HT22 cells death at 24 hours after reoxygenation, whereas DHM attenuated OGD-induced cell death (Fig. 5A, B). Moreover, OGD activated cleaved caspase-3 in HT22 cells, which was significantly inhibited by DHM in a dose-dependent manner (Fig. 5C). Taken together, our data therefore reveal that DHM protects neuronal cells through two pathways: one is to elicit direct protection on neuron via inhibition of cleaved caspase-3 activity, another is to inhibit microglial activation.

\subsection{DHM activates ERK1/2 and CREB in HT22 neuronal cells that may contribute to the protection}

To understand the signaling mechanism of DHM-elicited neuroprotection, we found that DHM alone increased the phosphorylation of ERK1/2 and CREB in HT22 cells in a time and dose-dependent manner (Fig. 6A, B). U0126, a selective inhibitor of MEK1/2, attenuated DHM-activated ERK1/2 and CREB (Fig. 6C). Further more, OGD/reoxygenation significantly inhibited phosphorylation of ERK1/2, CREB as well as Bcl-2 protein expression, while DHM treatment restored the reduced activation of ERK1/2, CREB and Bcl-2 protein expression (Fig. 7A). Consistently, application of U0126 to inhibit ERK1/2 and CREB activation also canceled the neuroprotection of DHM on OGD-R neurons (Fig. 7B).

\section{Discussion}

Ischemic stroke is one of the leading causes of death and disability. Following stroke, many pathological cascades were induced, including oxidative stress, inflammation, mitochondrial damage and excitotoxicity (Goldstein, 2011). Therefore, there is in urgent need to develop new strategies to treat ischemic stroke. We showed here that administration of DHM, a potential food supplement, protected against cerebral ischemia/reperfusion injury and functional recovery from stoke. The DHM elicits the beneficial effects via suppressed pro-inflammatory responses and direct neuroprotection. Moreover, our data revealed that the protection of DHM in HT22 cells were associated with ERK1/2, CREB and Bcl-2 signaling pathway.

DHM purified from Ampelopsis grossedentata that is widely distributed in southern 
China. Many present studies demonstrated DHM possesses anti-cancer, DHM induces mouse hepatoma Hepal-6 cell apoptosis via the TGF- $\beta$ signaling pathway. P53-related pathways may contribute to DHM-induced gastric cancer cells apoptosis. Interestingly, we found the treatment of DHM was protective for normal HT22 cells, which is distinctive compared with the effects of it stimulates apoptosis of cancer cells. Indeed, it is not uncommon that the contradictory data exist in the fields of cerebral ischemia and cancer research. For instance, cyclin-dependent kinases inhibition promotes cancer cell death but protects neuronal cells from ischemic injuries (Asghar, Witkiewicz, Turner, \& Knudsen, 2015; Sebastien Cagnol \& Chambard, 2009). The cell-specific death and surviving signaling associated with DMH under different pathological conditions needs further investigation in depth. For example, evidence exists to suggest that DHM exerts high radical scavenging and anti-oxidant activity, which may account for DMH neuroprotection against ischemic damage. .

Current evidence suggests that microglia-mediated neuroinflammation plays an important role in ischemic ischemia/reperfusion via the release of many pro-inflammatory mediators (Sharma \& Ganguly, 2003). Therefore, ischemic neuroinflammation is thought to be a valid target for stroke therapies. In the MCAO animal models, treatment with DHM significantly reduced the infarct volumes, microglial activation and attenuated behavioral impairments following ischemic brain injury (Fig. 1, 2). By using the BV2 microglial cell line, we observed that DHM significantly reduced LPS-induced NO production (Fig. 3B), inhibited the protein and mRNA expression levels of iNOS and COX-2 upregulation, and lowered secretion of IL-6 and TNF- $\alpha$ (Fig. 3C, 3D, 3E). It is possible that DHM exerts obvious effects of suppression neuroinflammation can be applied to other CNS diseases which companied with many proinflammatory mediators, such as $\mathrm{PD}, \mathrm{AD}$, depression or multiple sclerosis. Moreover, we demonstrated that DHM-inhibited microglial activation is contributed to the neuroprotection of DHM, since the conditioned medium from BV2 cells co-treated with DHM and LPS protected HT22 cells against apoptosis (Fig. 4). LPS-induced microglia/neuron systems can simulate microenvironments of some diseases. Our data suggested that DHM likely reduced 
ischemia-induced neuronal damage by suppressing microglial activation and the release of pro-inflammatory mediators.

In addition to inhibition on microglial activation, we also found that DHM directly protected against OGD-induced apoptosis. Compared to vehicle, DHM treatment increased cell viability of HT22 neuronal cells and attenuated the activation of caspase-3 following OGD. It is well known that ERK1/2 are members of the mitogen-activated protein kinases (MAPKs) family that regulate cell survival and apoptosis (Jia et al., 2008; Sawe, Steinberg, \& Zhao, 2008). The activated ERK1/2 further phosphorylates membrane and cytoplasm proteins. When translocating from cytosol to cell nucleus, it phosphorylates various transcription factors, such as the cAMP-response element binding protein, CREB (Bui et al., 2012; Screaton et al., 2004). CREB is a pro-survival protein that bound to DNA CRE element to regulate expression of a number of downstream genes. Phosphorylation of CREB upregulates a various of neuroprotective gene, such as BDNF, Bcl-2, PGC-1a and Bcl-XL (Réus et al., 2011; Yune, Park, Lee, \& Oh, 2008). Our results showed that treatment of DHM alone increased the phosphorylation of ERK1/2 and CREB in HT22 cells in a time and dose-dependent manner (Fig. 6A, B). Moreover, the effects of DHM were attenuated by the ERK1/2 inhibitor U0126, suggesting that DHM acted through the ERK1/2-CREB pathway (Fig. 6C). In the OGD model, we found that DHM significantly blunted decrease in phosphorylation of ERK1/2, CREB and Bcl-2 protein induced by OGD (Fig. 7A), inhibition of ERK1/2 and CREB activation abolished DHM-mediated neuroprotection following OGD (Fig. 7B). Although we did not examine that CREB-mediated Bcl-2 expression protected HT22 following OGD, previous publication have shown that CREB-mediated Bcl-2 expression are neuroprotective in the neuronal OGD model (Bui et al., 2012). Taken together, the study suggested that DHM modulates the ERK1/2-CREB-Bcl2 pathway, which may contribute to DHM protection following ischemic stroke.

In summary, our experimental results showed that DHM protected against cerebral ischemic injury possibly by suppressing microglia-mediated neuroinflammation and activating neuronal ERK-CREB-Bcl-2 signaling pathway. Given the wide protective 
effects of DHM against damages and insults (Linying et al., 2015), future clinical research may be needed to investigate whether DHM and its analogs may be a potential food supplement for treating ischemic stroke.

\section{Acknowledgments}

This work received the financial support of grants from the National Science

Foundation of China $(81373382,81571124,81372688$ and 81371278$)$ and the

Specialized Research Fund for the Doctoral Program of Higher Education of China

(20133201110017). Additionally, support from the Priority Academic Program

Development of the Jiangsu Higher Education Institutes (PAPD) and the Jiangsu key

laboratory grant (BM2013003) is appreciated.

\section{References}

Asghar, U., Witkiewicz, A. K., Turner, N. C., \& Knudsen, E. S. (2015). The history and future of targeting cyclin-dependent kinases in cancer therapy. Nat Rev Drug Discov, 14(2), 130-146. doi: $10.1038 / \mathrm{nrd} 4504$

Bui, C., Barter, M. J., Scott, J. L., Xu, Y., Galler, M., Reynard, L. N., . . . Young, D. A. (2012). cAMP response element-binding (CREB) recruitment following a specific CpG demethylation leads to the elevated expression of the matrix metalloproteinase 13 in human articular chondrocytes and osteoarthritis. Faseb Journal, 26(7), 3000-3011.

Cagnol, S., \& Chambard, J. C. (2010). ERK and cell death: mechanisms of ERK-induced cell death--apoptosis, autophagy and senescence. FEBS J, 277(1), 2-21. doi: 10.1111/j.1742-4658.2009.07366.x

Chen, Y., Zhang, L., Ni, J., Wang, X., Cheng, J., Li, Y., . . . Jia, J. (2016). LLDT-8 protects against cerebral ischemia/reperfusion injury by suppressing post-stroke inflammation. J Pharmacol Sci, 131(2), 131-137. doi: 10.1016/j.jphs.2016.05.003

Choi, I. Y., Ju, C., Anthony Jalin, A. M., Lee, D. I., Prather, P. L., \& Kim, W. K. (2013). Activation of cannabinoid CB2 receptor-mediated AMPK/CREB pathway reduces cerebral ischemic injury. Am J Pathol, 182(3), 928-939. doi: 10.1016/j.ajpath.2012.11.024

Denes, A., Vidyasagar, R., Feng, J., Narvainen, J., McColl, B. W., Kauppinen, R. A., \& Allan, S. M. (2007). Proliferating resident microglia after focal cerebral ischaemia in mice. J Cereb Blood Flow Metab, 27(12), 1941-1953. doi: 10.1038/sj.jcbfm.9600495

Fan, T. F., Wu, T. F., Bu, L. L., Ma, S. R., Li, Y. C., Mao, L., . . . Zhang, W. F. (2016). Dihydromyricetin promotes autophagy and apoptosis through ROS-STAT3 signaling in head and neck squamous cell carcinoma. Oncotarget, 7(37), 59691-59703. doi: 10.18632/oncotarget.10836. 
Feigin, V. L., Mensah, G. A., Norrving, B., Murray, C. J., Roth, G. A., \& Group, G. B. D. S. P. E. (2015). Atlas of the Global Burden of Stroke (1990-2013): The GBD 2013 Study. Neuroepidemiology, 45(3), 230-236. doi: 10.1159/000441106

Gaire, B. P., Kwon, O. W., Park, S. H., Chun, K. H., Kim, S. Y., Shin, D. Y., \& Choi, J. W. (2015). Neuroprotective effect of 6-paradol in focal cerebral ischemia involves the attenuation of neuroinflammatory responses in activated microglia. PLoS One, 10(3), e0120203. doi: 10.1371/journal.pone.0120203

Gan, P., Zhang, L., Chen, Y., Zhang, Y., Zhang, F., Zhou, X., . . . Zheng, L. T. (2015). Anti-inflammatory effects of glaucocalyxin B in microglia cells. J Pharmacol Sci, 128(1), 35-46. doi: 10.1016/j.jphs.2015.04.005

Goldstein. (2011). Guidelines for the Primary Prevention of Stroke: A Guideline for Healthcare Professionals From the American Heart Association/American Stroke Association (vol 42, pg 517, 2011). Stroke, 42(2), E26-E26.

Hacke, W., Bousser, M. G., Ford, G., Bath, P. M. W., Brainin, M., Caso, V., . . Al, E. (2008). Guidelines for the management of ischaemic stroke and transient ischaemic attack 2008. Stroke Practical Management Third Edition, 25(1).

Hou, X., Tong, Q., Wang, W., Xiong, W., Shi, C., \& Fang, J. (2015). Dihydromyricetin protects endothelial cells from hydrogen peroxide-induced oxidative stress damage by regulating mitochondrial pathways. Life Sciences, 130, 38-46.

Hou, X. L., Tong, Q., Wang, W. Q., Shi, C. Y., Xiong, W., Chen, J., . . Fang, J. G. (2015). Suppression of Inflammatory Responses by Dihydromyricetin, a Flavonoid from Ampelopsis grossedentata, via Inhibiting the Activation of NF-kappaB and MAPK Signaling Pathways. J Nat Prod, 78(7), 1689-1696. doi: 10.1021/acs.jnatprod.5b00275

Howard, R. (2010). Ischaemic stroke. Anaesthesia \& Intensive Care Medicine, 11(9), 340-342.

Jia, J., Chen, X., Zhu, W., Luo, Y., Hua, Z., \& Xu, Y. (2008). CART protects brain from damage through ERK activation in ischemic stroke. Neuropeptides, 42(5-6), 653-661. doi: 10.1016/j.npep.2008.05.006

Jiang, L., Zhang, Q., Ren, H., Ma, S., Lu, C., Liu, B., . . Zhu, R. (2015). Dihydromyricetin Enhances the Chemo-Sensitivity of Nedaplatin via Regulation of the $\mathrm{p53} / \mathrm{Bcl}-2$ Pathway in Hepatocellular Carcinoma Cells. PLoS One, 10(4), e0124994. doi: 10.1371/journal.pone.0124994

Jin, Q., Cheng, J., Liu, Y., Wu, J., Wang, X., Wei, S., . . Z Zhen, X. (2014). Improvement of functional recovery by chronic metformin treatment is associated with enhanced alternative activation of microglia/macrophages and increased angiogenesis and neurogenesis following experimental stroke. Brain Behav Immun, 40, 131-142. doi: 10.1016/j.bbi.2014.03.003

Linying, S., Ting, Z., Xinyu, L., Qin, H., Juan, H., Yong, Z., . . . Mantian, M. (2015). Dihydromyricetin improves skeletal muscle insulin resistance by inducing autophagy via the AMPK signaling pathway. Molecular \& Cellular Endocrinology, 409(C), 92-102.

Liu, B., Zhou, W., Chen, X., Xu, F., Chen, Y., Liu, J., . . Zhu, R. (2015). Dihydromyricetin induces mouse hepatoma Hepal-6 cell apoptosis via the transforming growth factor-beta pathway. Mol Med Rep, 11(3), 1609-1614. doi: 10.3892/mmr.2014.2891

Meschia, J. F., Bushnell, C., Boden-Albala, B., Braun, L. T., Bravata, D. M., Chaturvedi, S., ... Council on, H. (2014). Guidelines for the primary prevention of stroke: a statement for healthcare professionals from the American Heart Association/American Stroke Association. Stroke, 45(12), 3754-3832. doi: 10.1161/STR.0000000000000046 
Ni, J., Wang, X., Chen, S., Liu, H., Wang, Y., Xu, X., . . Z Zhen, X. (2015). MicroRNA let-7c-5p protects against cerebral ischemia injury via mechanisms involving the inhibition of microglia activation. Brain Behav Immun. doi: 10.1016/j.bbi.2015.04.014

Réus, G. Z., Stringari, R. B., Ribeiro, K. F., Ferraro, A. K., Vitto, M. F., Cesconetto, P., . . Quevedo, J. (2011). Ketamine plus imipramine treatment induces antidepressant-like behavior and increases CREB and BDNF protein levels and PKA and PKC phosphorylation in rat brain. Behavioural Brain Research, 221(1), 166-171.

Ren, Z. X., Zhao, Y. F., Cao, T., \& Zhen, X. C. (2016). Dihydromyricetin protects neurons in an MPTP-induced model of Parkinson's disease by suppressing glycogen synthase kinase-3 beta activity. Acta Pharmacol Sin, 37(10), 1315-1324. doi: 10.1038/aps.2016.42

Sasaki, T., Takemori, H., Yagita, Y., Terasaki, Y., Uebi, T., Horike, N., . . Kitagawa, K. (2011). SIK2 is a key regulator for neuronal survival after ischemia via TORC1-CREB. Neuron, 69(1), 106-119. doi: 10.1016/j.neuron.2010.12.004

Sawe, N., Steinberg, G., \& Zhao, H. (2008). Dual roles of the MAPK/ERK1/2 cell signaling pathway after stroke. Journal of Neuroscience Research, 86(8), 1659-1669. doi: 10.1002/jnr.21604

Screaton, R. A., Conkright, M. D., Katoh, Y., Best, J. L., Canettieri, G., Jeffries, S., . . Takemori, H. (2004). The CREB coactivator TORC2 functions as a calcium- and CAMP-sensitive coincidence detector. Cell, 119(1), 61-74.

Sharma, M., \& Ganguly, N. K. (2003). Release of pro-inflammatory mediators during myocardial ischemia/reperfusion in coronary artery bypass graft surgery. Molecular and Cellular Biochemistry, 247(1), 23-30.

Shi, L., Zhang, T., Zhou, Y., Zeng, X., Ran, L., Zhang, Q., . . . Mi, M. (2015). Dihydromyricetin improves skeletal muscle insulin sensitivity by inducing autophagy via the AMPK-PGC-1alpha-Sirt3 signaling pathway. Endocrine, 50(2), 378-389. doi: 10.1007/s12020-015-0599-5

Tao, L., Zhang, F., Hao, L., Wu, J., Jia, J., Liu, J.-y., . . . Zhen, X. (2014). 1-O-Tigloyl-1-O-deacetyl-nimbolinin B Inhibits LPS-Stimulated Inflammatory Responses by Suppressing NF-KB and JNK Activation in Microglia Cells. Journal of Pharmacological Sciences, 125(4), 364-374. doi: 10.1254/jphs.14025FP

Taylor, R. A., \& Sansing, L. H. (2013). Microglial responses after ischemic stroke and intracerebral hemorrhage. Clin Dev Immunol, 2013, 746068. doi: 10.1155/2013/746068

Wang, Y., Guo, L., Jiang, H., Zheng, L., Zhang, A., \& Zhen, X. (2016). Allosteric Modulation of Sigma-1 Receptors Elicits Rapid Antidepressant Activity. CNS Neurosci Ther. 22(5), 368-77 doi: 10.1111/cns.12502

Weinstein, J. R., Koerner, I. P., \& Moller, T. (2010). Microglia in ischemic brain injury. Future Neurol, 5(2), 227-246. doi: 10.2217/fnl.10.1

Wu, Z., Li, L., Zheng, L. T., Xu, Z., Guo, L., \& Zhen, X. (2015). Allosteric modulation of sigma-1 receptors by SKF83959 inhibits microglia-mediated inflammation. J Neurochem, 134(5), 904-914. doi: 10.1111/jnc.13182

Ye, L., Wang, H., Duncan, S. E., Eigel, W. N., \& O'Keefe, S. F. (2015). Antioxidant activities of Vine Tea (Ampelopsis grossedentata) extract and its major component dihydromyricetin in soybean oil and cooked ground beef. Food Chem, 172, 416-422. doi: 10.1016/j.foodchem.2014.09.090

Yujiri, T., Sather, S., Fanger, G. R., \& Johnson, G. L. (1998). Role of MEKK1 in Cell Survival and Activation of JNK and ERK Pathways Defined by Targeted Gene Disruption. Science, 282(5395), 1911-1914. 
Yune, T. Y., Park, H. G., Lee, J. Y., \& Oh, T. H. (2008). Estrogen-induced Bcl-2 expression after spinal cord injury is mediated through phosphoinositide-3-kinase/Akt-dependent CREB activation. Journal of Neurotrauma, 25(9), 1121-1131.

\section{Figure 1}

A

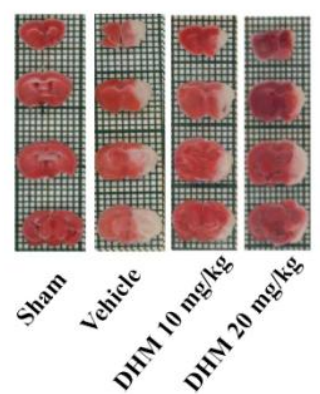

C

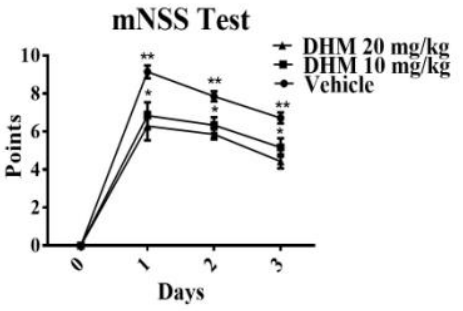

B

D

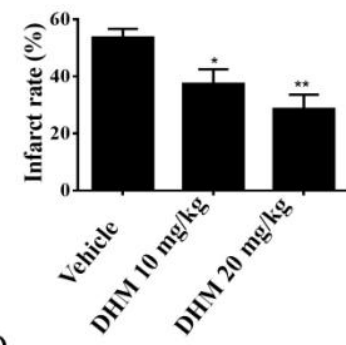

E

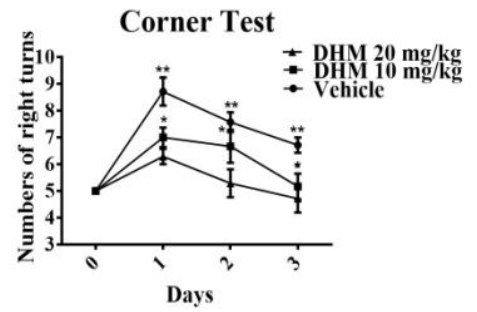

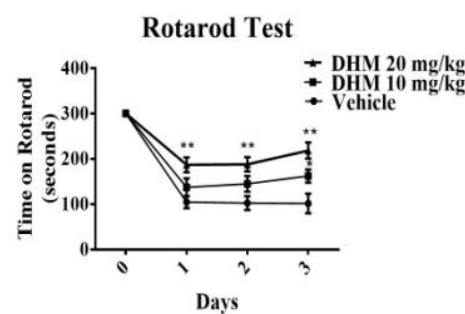


Figure 2

A

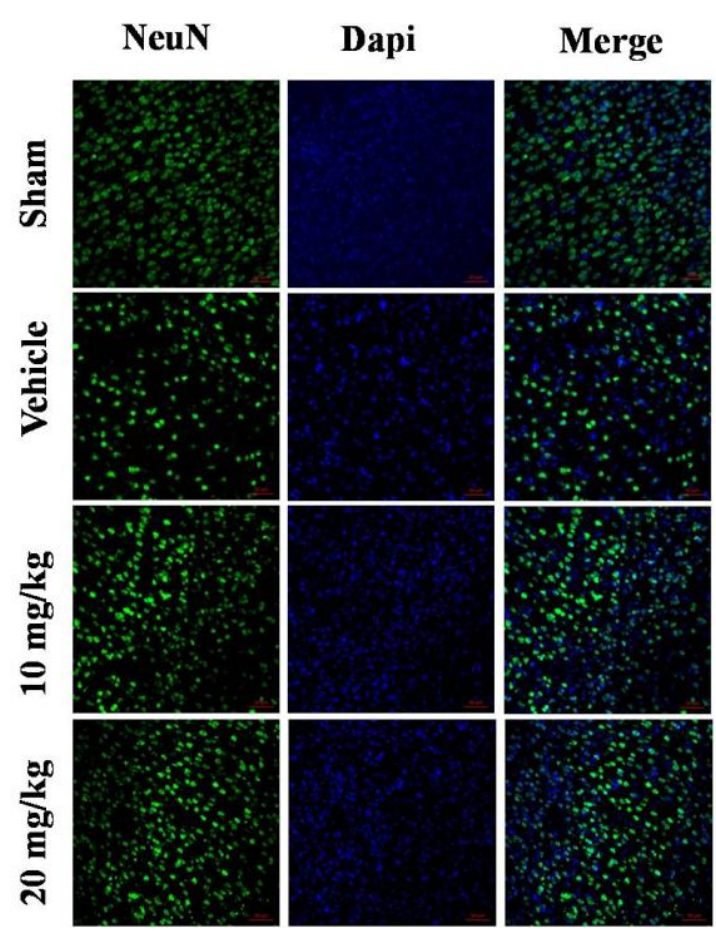

B

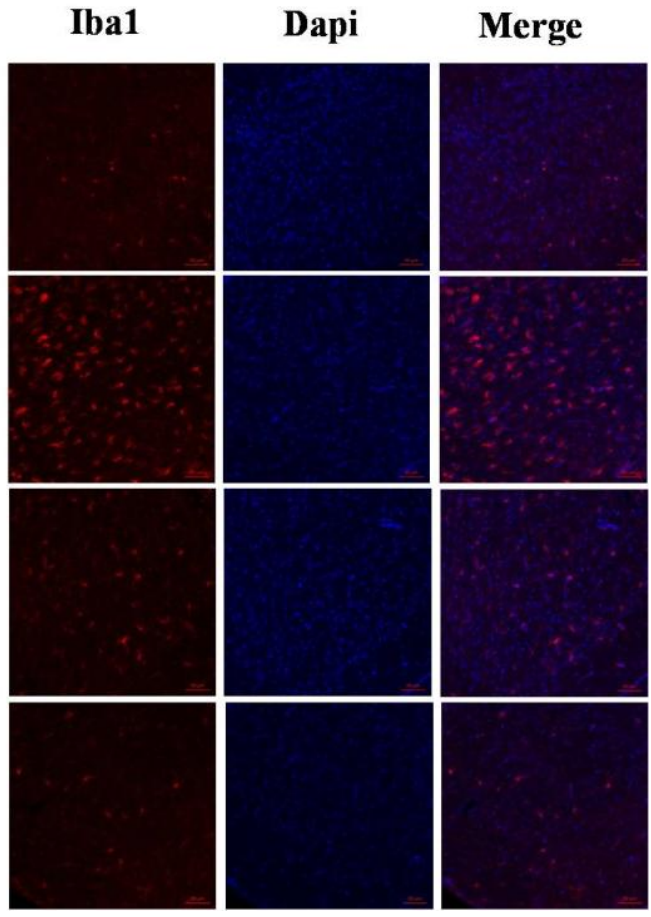


Figure 3

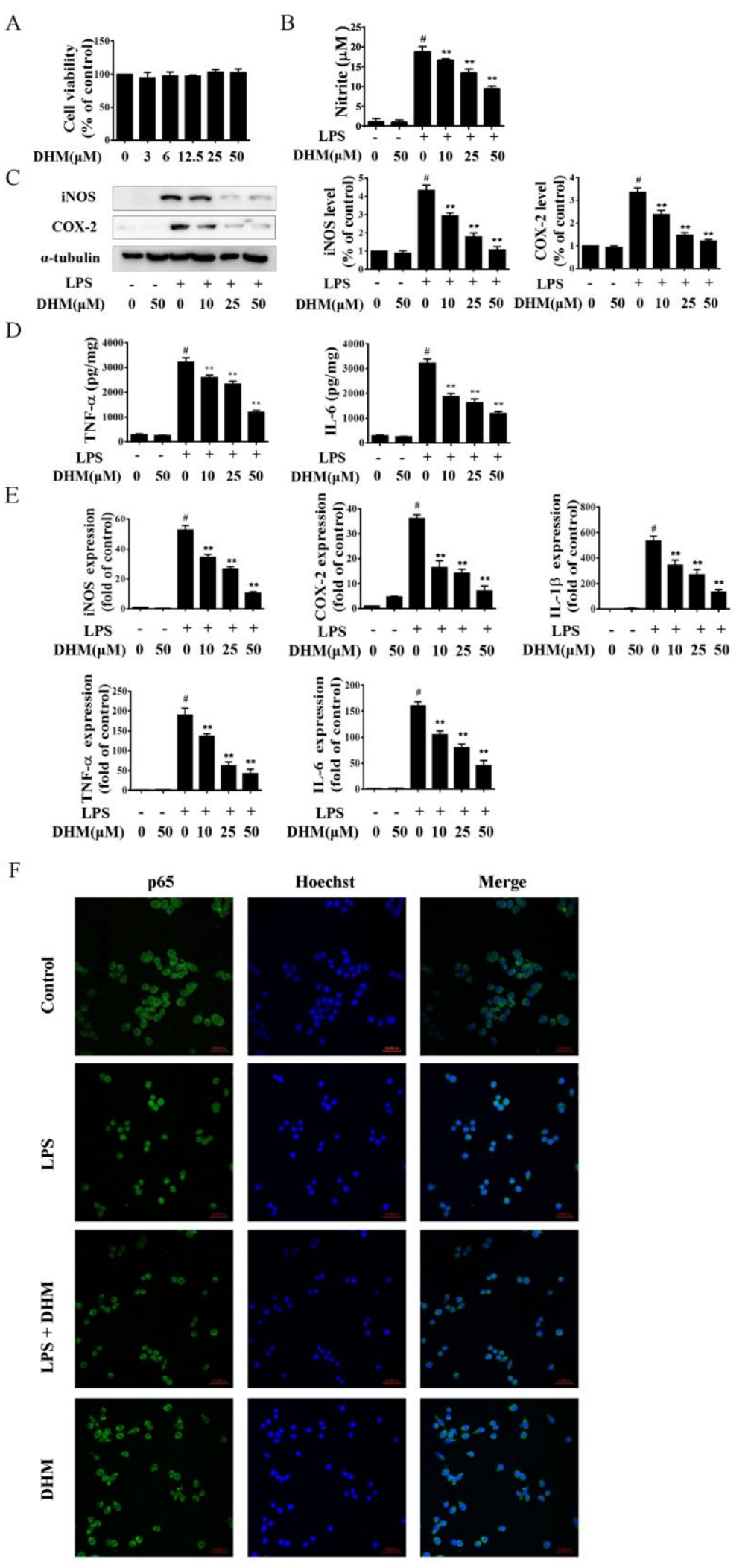


Figure 4
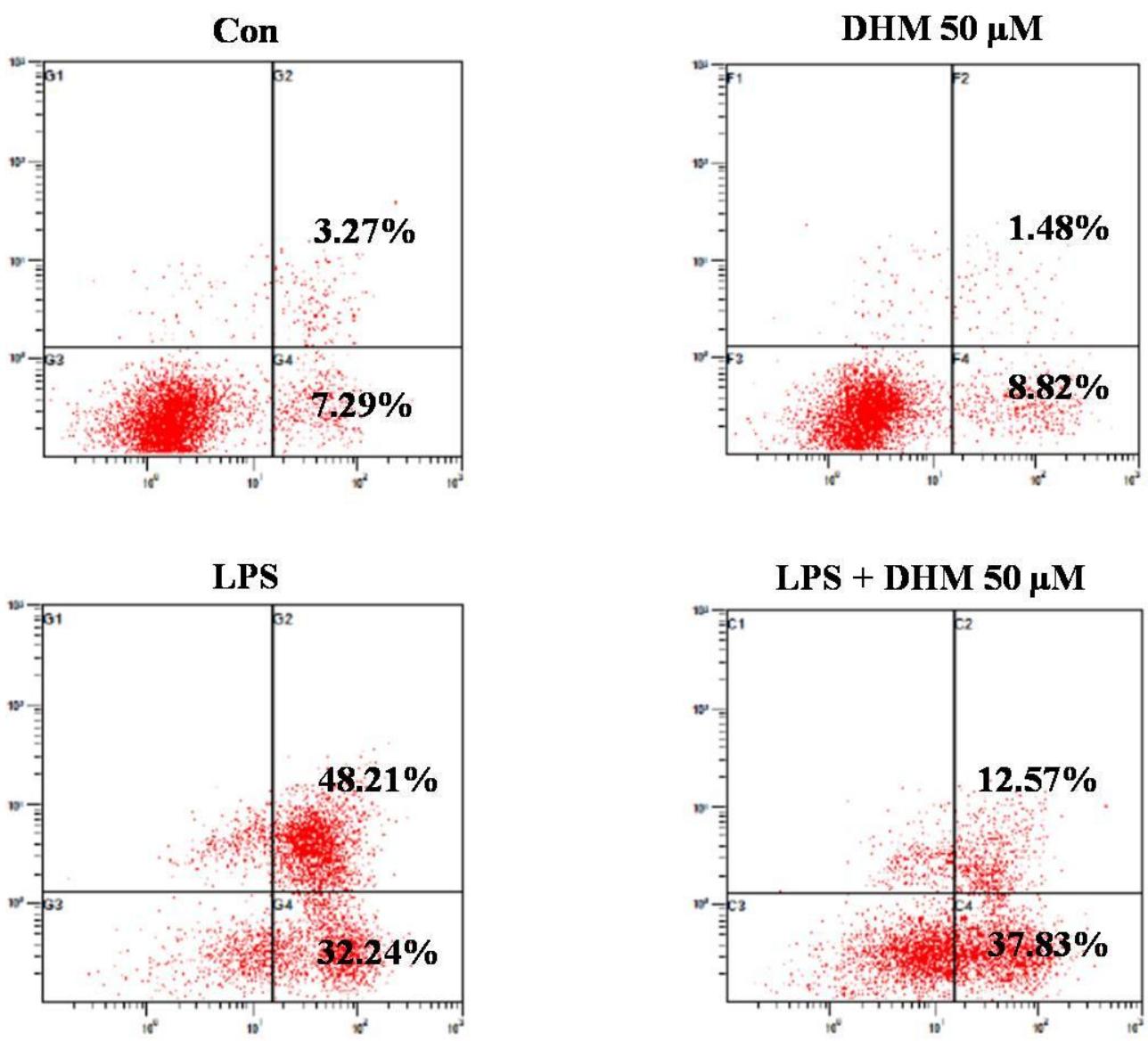
Figure 5

A

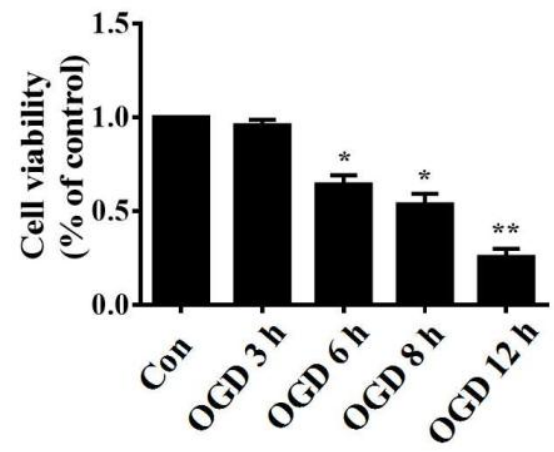

B
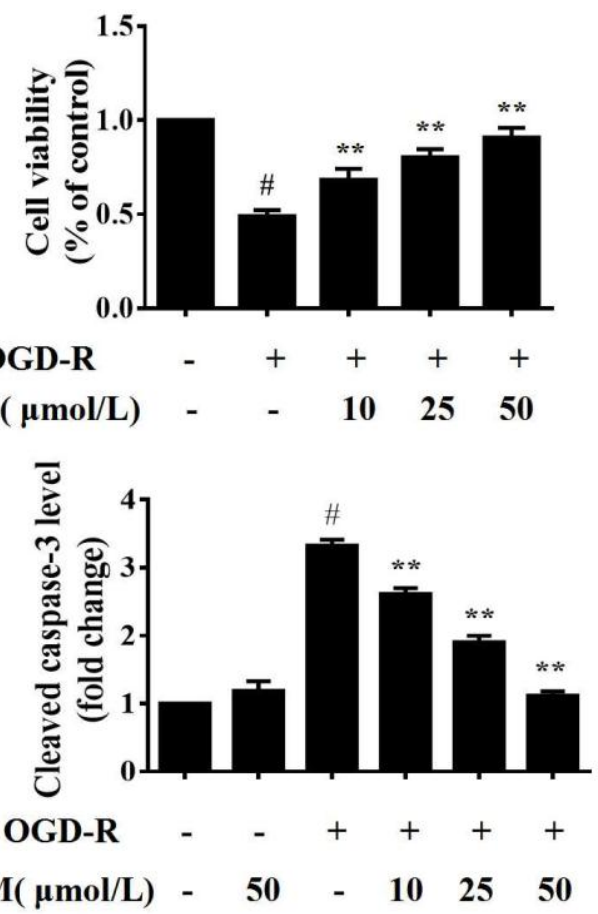

Figure 6

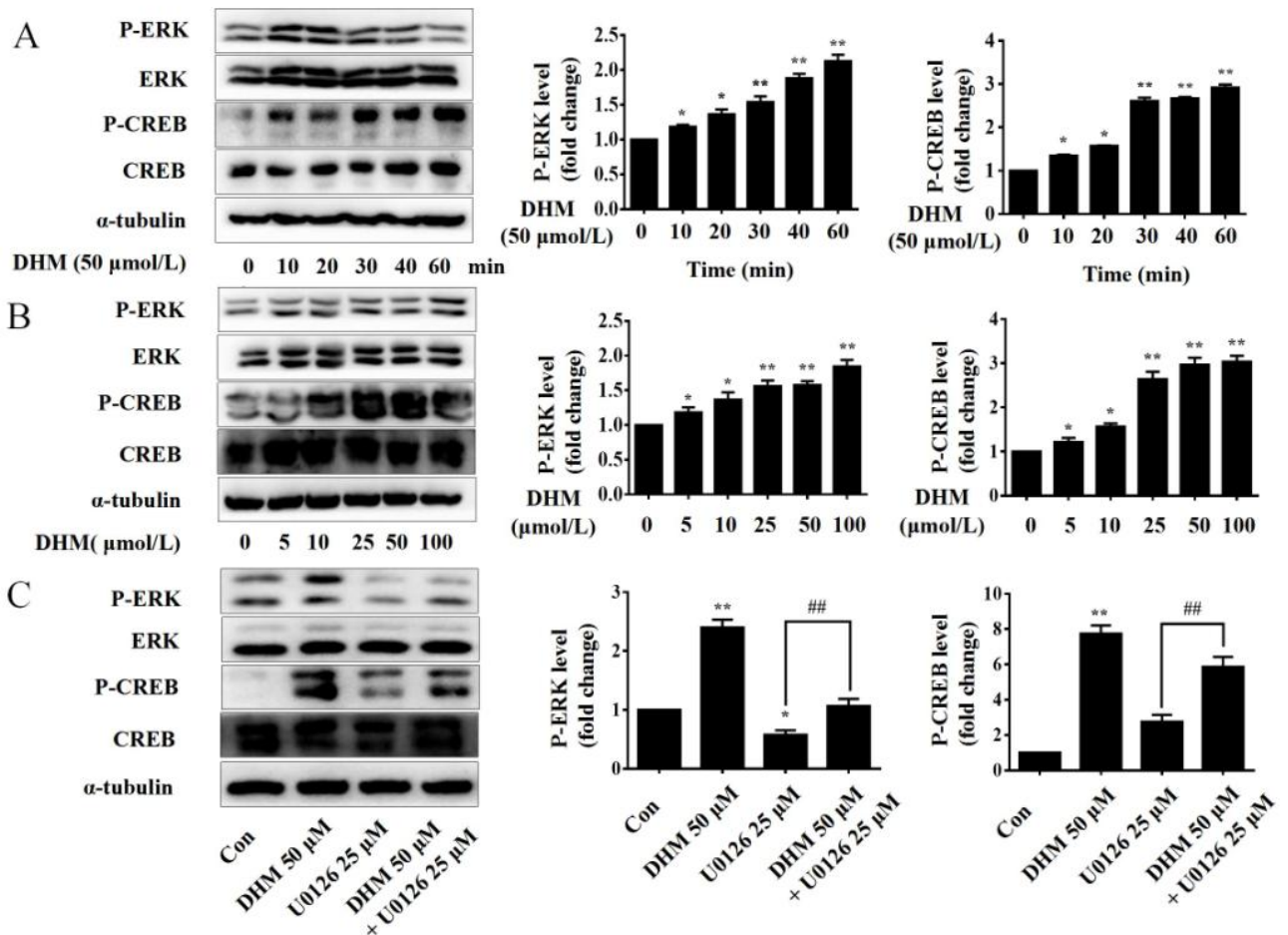




\section{Figure 7}

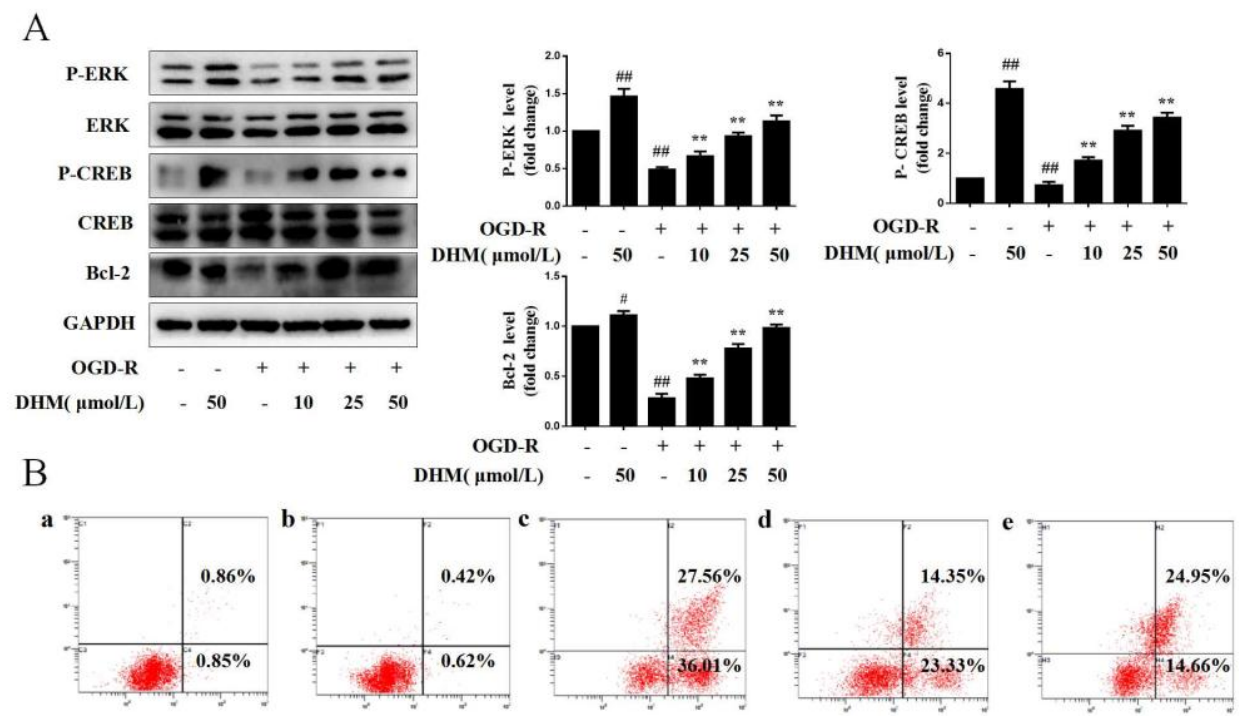

\section{Figure Legends}

Fig.1. DHM reduced brain ischemic damage and improved neurological deficits following. A) Representative images of 2,3,5-triphenyltetrazolium chloride (TTC) staining. The neuroprotective effects of DHM (10 mg/kg or $20 \mathrm{mg} / \mathrm{kg})$ were evaluated with TTC histology. B) Quantitative results of infarction volumes ( $\mathrm{n}=10$ per group) . C) Modified neurological severity scores (mNSS) were significantly lower in DHM-treated $(10 \mathrm{mg} / \mathrm{kg}$ or $20 \mathrm{mg} / \mathrm{kg})$ mice than those in vehicle-treated mice at 3 days after MCAO ( $\mathrm{n}=10$ per group). D) In the corner test, DHM improved sensorimotor asymmetric abnormalities of mice at 3 days after MCAO compared to vehicle group ( $\mathrm{n}=10$ per group). E) DHM-treated $(10 \mathrm{mg} / \mathrm{kg}$ or $20 \mathrm{mg} / \mathrm{kg}$ ) mice remained on the rotarod longer than the vehicle-treated mice following MCAO ( $\mathrm{n}=$ 10 per group).

Fig.2. DHM reduced microglial activation and neuron death in mice following MCAO. Microglia and Neuron were assessed 3 days after reperfusion by 
immunohistochemistry using microglial marker Iba1 and neuronal marker NeuN. A) The number of NeuN-positive cells (green) in ischemic core regions was increased in DHM-treated $(10 \mathrm{mg} / \mathrm{kg}, 20 \mathrm{mg} / \mathrm{kg})$ mice than the saline-treated mice following MCAO. B) Iba1-positive cells were reduced in ipsilateral cortex of DHM-treated mice vs. saline-treated mice following MCAO. Scale bars, $50 \mu \mathrm{m}$ (bottom panels)

Fig.3. The suppressive effects of DHM on the protein and mRNA expression of the inflammatory mediators and translocation of NF-kB (p65) in LPS-treated BV2 microglia. A) Cell viability measured using MTT assay. B) The nitric oxide (NO) levels assessed with Griess assay. C) The protein expression levels of iNOS, COX-2 were examined with western blotting. D) The protein expression levels of TNF- $\alpha$ and IL-6 were measured by ELISA. E) The mRNA expression levels of iNOS, COX-2, IL-6, IL-1 $\beta$, TNF- $\alpha$ were assessed with q-PCR. $\mathrm{n}=3$. * $\mathrm{P}<0.05, * * \mathrm{P}<0.01$, compared to LPS treated alone; \# P $<0.01$, compared to control; F) Immunocytochemistry results showed that DHM inhibited the translocation of NF- $\kappa B$ (p65) in LPS-treated BV2 microglia.

Fig.4. DHM inhibited apoptosis of HT22 neuronal cells induced by conditioned medium collected from LPS-activated BV2 microglia. The percentage of apoptotic cells was measured by flow cytometry.

Fig.5. DHM protected HT22 cells from OGD/ reoxygenation-induced cytotoxicity. Cell viability was assessed by the MTT assay. A) Cell viability of HT22 cells exposed to different duration of OGD followed by $24 \mathrm{~h}$ of reoxygenation. B) Cell viability of HT22 cells subjected to OGD/ reoxygenation and treated with different concentrations of DHM. C) The expression levels of cleaved (activated) caspase-3 analyzed by Western blotting. $\mathrm{n}=3$. $* \mathrm{P}<0.05, * * \mathrm{P}<0.01$, compared to OGD-R; \# $\mathrm{P}<0.01$, compared to control.

Fig.6. The effect of DHM on the phosphorylation of ERK1/2, CREB in a time and 
dose-dependent manner and conteract the effect of U0126. A) Time course of phosphorylation of ERK1/2 and CREB in HT22 cells treated with $50 \mu \mathrm{M}$ of DHM alone. B) Phosphorylation of ERK1/2 and CREB in HT22 cells treated with different concentrations of $\operatorname{DHM}(0,10,25,50,100 \mu \mathrm{mol} / \mathrm{L})$. C) The selective ERK1/2 inhibitor U0126 suppressed DHM-upregulated phosphorylation of ERK1/2 and CREB in HT22 cells. $\mathrm{n}=3$. * $\mathrm{P}<0.05, * * \mathrm{P}<0.01$, compared to control; \# $\mathrm{P}<0.01$, compared to treated-U0126.

Fig.7. The effect of DHM on regulation of the phosphorylation of ERK1/2 and CREB and protein expression of Bcl-2 and on apoptosis rates in HT22 cells after OGD/ reoxygenation A) HT22 cells were treated with different concentrations of DHM (10 $\mu \mathrm{M}, 25 \mu \mathrm{M}, 50 \mu \mathrm{M})$ and exposed to $8 \mathrm{~h}$ of OGD followed by reperfusion. $\mathrm{n}=3$. ${ }^{* *} \mathrm{P}$ $<0.01$, compared to control; \# P < 0.01, compared to OGD-R. B) Co-treatment with $50 \mu \mathrm{M}$ of DHM and $25 \mu \mathrm{M}$ U0126 did not inhibit apoptosis in HT22 cells after OGD/R. a. normal HT22 cells, b. cells treated with $50 \mu \mathrm{M}$ DHM, c. cells only exposed to OGD/R, d. cells treated with $50 \mu \mathrm{M}$ DHM during OGD/R, e. cells treated with $50 \mu \mathrm{M}$ DHM and $25 \mu \mathrm{M}$ U0126 during OGD/R. The percentage of apoptotic cells was measured by flow cytometry. 